\title{
Correção de solos salino-sódicos pela aplicação de gesso mineral ${ }^{1}$
}

\author{
Ronaldo M. Melo², Maria de F. C. Barros², Paulo M. dos Santos² \& Mário M. Rolim²
}

\begin{abstract}
RESUMO
Com o objetivo de quantificar os parâmetros que determinam a efetividade do gesso na recuperação de solos salinosódicos do perímetro irrigado de Custódia, localizado em Pernambuco, visando ao aumento das áreas disponíveis para a agricultura irrigada um experimento foi realizado usando-se colunas de solo instaladas no laboratório de Mecânica do Solo e Aproveitamento de Resíduos da Universidade Federal Rural de Pernambuco. Os tratamentos foram dispostos em delineamento em blocos casualizados com arranjo fatorial de dois solos e seis níveis de gesso de jazida, equivalentes a $25,50,75,100,125$ e 150\% da necessidade de gesso, determinados pelo método de laboratório Schoonover M-1, incorporados aos primeiros $5 \mathrm{~cm}$ da coluna de solo com três repetições. Os componentes avaliados foram a condutividade elétrica, a relação de adsorção de sódio (RAS) e os cátions solúveis do extrato da pasta saturada. O nível equivalente a 100\% da necessidade de gesso mostrou-se eficaz na diminuição da RAS do extrato de saturação dos solos, reduzindo os teores de sódio dos solos.
\end{abstract}

Palavras-chave: condutividade elétrica, sodicidade, RAS

\section{Reclamation of saline-sodic soils by application of mineral gypsum}

\begin{abstract}
With the objective of quantifying the parameters that determine the effectiveness of gypsum in the reclamation of salinesodic soils in the Irrigated Perimeter of Custódia located in the State of Pernambuco, Brazil, with the purpose of increasing the available areas for irrigated agriculture, an experiment was carried out in soil columns installed at the Soil Mechanics and Residue Recovery Laboratory at the Universidade Federal Rural de Pernambuco. The treatments were arranged in a randomized blocks and a factorial scheme of two soils and six levels of mined gypsum equivalent to 25, 50, 75, 100, 125 and $150 \%$ of gypsum requirements of the soil determined by Schoonover M-1 laboratory method, incorporated to the first $5 \mathrm{~cm}$ of the soil column, with three replications. The evaluated components were the electrical conductivity, the sodium adsorption ratio (SAR) and the soluble cations of the saturation extract. The level of gypsum equivalent to $100 \%$ of the gypsum requirement showed to be most effective at reducing the SAR of the extract, reducing soil contents of sodium in the soil.
\end{abstract}

Key words: electrical conductivity, sodicity, SAR

Parte da Dissertação de Mestrado do primeiro autor, apresentada à Universidade Federal Rural de Pernambuco

2 UFRPE, CEP 52171-900. Recife, PE. Fone: (81) 3320-6222. E-mail: agroronaldo@hotmail.com; fatima@depa.ufrpe.br; santos.p.m@bol.com.br; rolim@dtr.ufrpe.br 


\section{INTRODUÇÃO}

Solos afetados por sais contêm sais solúveis e/ou sódio trocável em quantidades suficientes para reduzir ou interferir no desenvolvimento vegetal e, conseqüentemente, na produção das culturas. Sendo esta uma das limitações da produção agrícola mundial, sobretudo em áreas irrigadas localizadas em zonas áridas e semi-áridas.

A maior parte do perímetro irrigado de Custódia encontra-se com problemas de sais e suas atividades agrícolas praticamente cessaram devido aos problemas de salinidade e sodicidade (Oliveira et al., 2002).

A necessidade de se produzir alimentos impõe a recuperação e o manejo dessas áreas. A recuperação de solos afetados por sais tem por objetivo a redução da concentração dos sais solúveis e do sódio trocável no perfil do solo.

Vários corretivos podem ser utilizados na recuperação de solos com excesso de sódio trocável, como gesso, enxofre, sulfato de alumínio, cloreto de cálcio e ácido sulfúrico; entretanto, o gesso é o produto mais comumente usado em razão do seu baixo custo e abundância com que é encontrado na maior parte do mundo. No território brasileiro, as principais reservas ocorrem associadas às bacias sedimentares entre as quais a do Araripe, que abrange os estados do Piauí, Ceará e Pernambuco, sendo este o pólo mais produtivo de gesso do País, denominado pólo gesseiro do Araripe (Nascimento \& Barros, 2003; Barros et al., 2004).

Em geral, a quantidade de gesso é determinada por métodos de laboratório, em que método de Schoonover (1952) é utilizado como padrão (Richards, 1954). Segundo Barros \& Magalhães (1989) sais solúveis presentes no solo interferem no sistema, subestimando a necessidade de gesso do solo determinada segundo o procedimento de Schoonover. Os autores apresentaram uma modificação nesta metodologia (Schoonover M-1) para os solos salino-sódicos de Pernambuco.

Sabendo-se que a salinização e/ou sodificação do solo é responsável pela redução na produção agrícola, culminando quase sempre com o abandono de áreas agricultáveis e que o alijamento dessas áreas acarreta grandes prejuízos à economia regional, a importância da recuperação desses solos é imprescindível para que os mesmos sejam reincorporados ao sistema de produção.

Objetivou-se, com o presente trabalho, avaliar o efeito da aplicação de gesso determinado pelo método de laboratório Schoonover M-1 nas características químicas dos solos salino-sódicos.

\section{MATERIAL E MÉTODOS}

O experimento foi conduzido no Laboratório de Mecânica do Solo e Aproveitamento de Resíduos, no Departamento de Tecnologia Rural da Universidade Federal Rural de Pernambuco, para este estudo foram coletadas duas amostras de solo Neossolo Flúvico salino-sódico, na profundidade de 0-40 cm, do perímetro de Custódia, PE.

A caracterização do solo foi realizada em amostras secadas ao ar, destorroadas e passadas em peneira com abertura de $2 \mathrm{~mm}$ de malha, retirando-se, antes desse procedimento, torrões, para obtenção da densidade do solo. O extrato de saturação foi obtido segundo metodologia descrita por Richards (1954); determinaram-se, no extrato da pasta saturada, a condutividade elétrica e os cátions e ânions solúveis: cálcio e magnésio por absorção atômica, sódio e potássio por fotometria de chama, carbonato e bicarbonato por titulação com $\mathrm{H}_{2} \mathrm{SO}_{4} 0,005 \mathrm{~mol} \mathrm{~L}^{-1}$ e cloreto por titulação com $\mathrm{AgNO}_{3} 0,025 \mathrm{~mol} \mathrm{~L}^{-1}$. O sulfato foi determinado por precipitação com cromato de bário, conforme a metodologia descrita por Magalhães (1987). A RAS (relação de adsorção de sódio) foi calculada pela expressão $\mathrm{RAS}=\mathrm{Na}^{+}$/ $\left[\left(\mathrm{Ca}^{+2}+\mathrm{Mg}^{+2}\right) / 2\right]^{1 / 2}$ e os resultados se encontram na Tabela 1.

Utilizou-se, para a determinação da necessidade de gesso (NG), uma modificação do método de laboratório de Schoonover (1952), Schoonover M-1, desenvolvida por Barros \& Magalhães (1989) para solos salino-sódicos de Pernambuco.

Aplicando-se a metodologia sugerida pela EMBRAPA (1997), na caracterização física, fizeram-se as análises da condutividade hidráulica pelo método descrito para solos com estrutura deformada com carga hidráulica constante, da composição granulométrica após lavagem com etanol a $60 \%$ até eliminação total de cloretos, da densidade das partículas pelo método do balão volumétrico e da densidade do solo através do método do torrão parafinado (Tabela 2).

As unidades experimentais foram constituídas de tubos plásticos de PVC com $10 \mathrm{~cm}$ de diâmetro e $20 \mathrm{~cm}$ de altura tendo, em sua base, um sistema de drenagem. As colunas de PVC receberam três camadas de solo, cada uma com $5 \mathrm{~cm}$ de espessura acondicionadas de modo a se aproximarem da densidade do solo.

Os tratamentos foram dispostos em um delineamento experimental em blocos ao acaso com um arranjo fatorial entre 6 níveis da necessidade de gesso $(25,50,75,100,125$ e 150\%) e duas amostras de solo salino-sódico (S1 e S2), com três repetições, totalizando 36 unidades experimentais. O gesso foi incorporado aos primeiros $5 \mathrm{~cm}$ do solo, antes do acondicionamento na coluna. Após aplicação do corretivo as colunas de solo foram umedecidas lentamente com água destilada até atingirem a saturação, permanecendo nesta umidade durante $24 \mathrm{~h}$, para restabelecimento do equilíbrio do sistema. Para evitar perdas por evaporação as colunas foram cobertas com sacos plásticos; em seguida, as colunas de solo foram lixiviadas com água destilada mantendo-se um nível constante de $2 \mathrm{~cm}$ acima da superfície do solo. Na lixiviação se utilizou uma lâmina de água equivalente a três vezes o volume de poros (1200 mL), dividida em quatro frações de $300 \mathrm{~mL}$ aplicadas aos $8,16,24$ e 32 dias após o período de saturação; depois da lixiviação as colunas foram desmontadas e os solos de cada camada secados ao ar, destorroados e passados em peneira de $2 \mathrm{~mm}$ de abertura; determinaram-se, no extrato da pasta saturada, os cátions solúveis e a condutividade elétrica (CE) de acordo com a metodologia descrita anteriormente.

Utilizou-se, nas análises estatísticas, o programa SAS versão 8.0, e os dados obtidos foram submetidos a análise de variância e de regressão, testando-se diversos modelos. Os critérios para escolha do modelo, foram o maior valor do coeficiente de determinação ajustado e a significância dos coeficientes da equação de regressão. 
Tabela 1. Composição do extrato da pasta saturada dos solos

\begin{tabular}{|c|c|c|c|c|c|c|c|c|c|c|c|}
\hline \multirow{2}{*}{ Amostra } & $\mathrm{Ca}^{2+}$ & $\mathrm{Mg}^{2+}$ & $\mathrm{Na}^{+}$ & $\mathrm{K}^{+}$ & $\overline{\mathrm{Cl}^{-}}$ & $\mathrm{SO}_{4}{ }^{2-}$ & $\mathrm{CO}_{3}{ }^{2-}$ & $\mathrm{HCO}_{3}{ }^{\circ}$ & \multirow{2}{*}{$\begin{array}{l}C^{(1)} \\
d S m^{-1}\end{array}$} & \multirow{2}{*}{$\begin{array}{c}\operatorname{RAS}^{(2)} \\
\left(\mathrm{mmol} \mathrm{L}^{-1}\right)^{1 / 2}\end{array}$} & \multirow{2}{*}{$\begin{array}{c}\mathrm{NG}^{(3)} \\
\mathrm{cmol}_{\mathrm{c}} \mathrm{dm}^{-3}\end{array}$} \\
\hline & \multicolumn{8}{|c|}{$\mathrm{mmol}_{\mathrm{c}} \mathrm{L}^{-1}$} & & & \\
\hline S1 & 50,26 & 18,17 & 148,52 & 0,64 & 192,2 & 25,79 & 0,00 & 1,85 & 22,4 & 25,39 & 3,12 \\
\hline S2 & 25,81 & 10,17 & 182,26 & 0,55 & 186,5 & 12,50 & 0,00 & 1,90 & 20,2 & 42,97 & 5,57 \\
\hline
\end{tabular}

(1) $\mathrm{CE}=$ condutividade elétrica do extrato da pasta saturada; (2) RAS = relação de adsorção de sódio e ${ }^{(3)} \mathrm{NG}=$ necessidade de gesso

Tabela 2. Características físicas das amostras de solos

\begin{tabular}{|c|c|c|c|c|c|c|c|c|}
\hline \multirow{3}{*}{ Amostra } & \multicolumn{3}{|c|}{ Análise Granulométrica } & \multirow{3}{*}{ Classe Textural } & \multirow{3}{*}{$\begin{array}{c}\text { Condutividade } \\
\text { Hidráulica } \\
\text { cm h h}^{-1}\end{array}$} & (1) $\mathrm{n}_{\mathrm{n}}$ & (2) & \multirow{3}{*}{$\begin{array}{l}\text { (3) PT } \\
\mathbf{m}^{3} \mathbf{m}^{-}\end{array}$} \\
\hline & Areia & Silte & Argila & & & (1)Dp & (2)Ds & \\
\hline & & $\%$ & & & & \multicolumn{2}{|c|}{$\mathrm{g} \mathrm{cm}^{-3}$} & \\
\hline S1 & 72,5 & 9,4 & 17,9 & Franco arenoso & 0,00 & 2,61 & 1,60 & 0,39 \\
\hline S2 & 60,4 & 15,7 & 23,9 & Franco-argilo-arenoso & 0,00 & 2,52 & 1,51 & 0,40 \\
\hline
\end{tabular}

(1) Dp - densidade das partículas; (2) Ds - densidade do solo e (3) PT - porosidade total

\section{RESULTADOS E DISCUSSÃO}

Os dados obtidos para CE encontram-se na Tabela 3. A utilização de gesso como corretivo de solos salino-sódicos e a aplicação de uma lâmina lixiviação equivalente a três volume de poros foram suficientes para permitir a retirada da maior parte dos sais solúveis das amostras de solo em todos os tratamentos. A CE dos solos, que era originalmente de 22,40 e $20,20 \mathrm{dS} \mathrm{m}^{-1}$, reduziu para valores máximos de 2,18 a $3,16 \mathrm{dS} \mathrm{m}^{-1}$ para os solos 01 e 02 , respectivamente, cujos resultados ocorreram, provavelmente, em virtude da aplicação do gesso aumentar, de inicio, a quantidade de sais no solo ocasionando a diminuição da dupla camada difusa enquanto a substituição do sódio trocável pelo cálcio floculou as argilas dispersas melhorando a condutividade hidráulica e, em conseqüência, a lixiviação dos sais solúveis; comportamento semelhante foi obtido por Gheyi et al. (1995) ao aplicarem gesso em um solo salino-sódico do perímetro irrigado de Sumé, na Paraíba, que constataram aumento da infiltração de água no solo em função da substituição do sódio trocável pelo cálcio do corretivo.

Verifica-se também, na Tabela 3, que as aplicações dos níveis de gesso e das lâminas de lixiviação reduziram os teores de magnésio, potássio, cálcio e sódio solúvel em relação aos teores originais das amostras de solo.

Os resultados obtidos para magnésio se devem, provavelmente, à substituição do magnésio trocável pelo cálcio do corretivo e lixiviação do mesmo pelas lâminas aplicadas. Segundo Barros et al. (2004), as pequenas quantidades de magnésio existentes são devidas, sem dúvida, à contribuição do gesso, que tem o magnésio como impureza na sua composição.

As menores concentrações encontradas para potássio são explicadas pela fácil lixiviação deste elemento, que é monovalente, e apresenta ligações mais fracas que o cálcio e o magnésio. Também se constata um acréscimo da concentração de cálcio solúvel a medida em que o nível de gesso aplicado aumentava; para a concentração de sódio observa-se comportamento inverso.

Em relação às camadas, sempre que aumentava a profundidade, o teor de cálcio diminuía e o de sódio aumentava indicando que, provavelmente, a menor concentração de cálcio nas camadas inferiores se deve à utilização de cálcio na substituição do sódio trocável da camada superior, resultados que
Tabela 3. Composição do extrato da pasta saturada depois da aplicação do gesso e lixiviação das colunas de solo, para as amostras de solos 1 e 2

\begin{tabular}{|c|c|c|c|c|c|c|c|}
\hline \multirow{2}{*}{ Solo } & $G^{(1)}$ & $\mathrm{Ca}^{2+}$ & $\mathrm{Mg}^{2+}$ & $\mathrm{Na}^{+}$ & $\mathrm{K}^{+}$ & \multirow{2}{*}{$\begin{array}{c}C E^{(2)} \\
d S m^{-1}\end{array}$} & \multirow{2}{*}{$\begin{array}{c}\operatorname{RAS}^{(3)} \\
\left(\mathrm{mmol} \mathrm{L}^{-1}\right)^{1 / 2}\end{array}$} \\
\hline & $\%$ & \multicolumn{4}{|c|}{$\mathrm{mmol}_{\mathrm{c}} \mathrm{L}^{-1}$} & & \\
\hline \multirow{6}{*}{$\begin{array}{c}1 \\
0-5 \mathrm{~cm}\end{array}$} & 25 & 1,87 & 0,64 & 19,29 & 0,13 & 2,14 & 17,10 \\
\hline & 50 & 1,99 & 0,64 & 17,84 & 0,13 & 2,11 & 15,47 \\
\hline & 75 & 2,36 & 0,75 & 17,15 & 0,15 & 2,11 & 13,75 \\
\hline & 100 & 4,22 & 0,61 & 10,85 & 0,11 & 1,94 & 6,98 \\
\hline & 125 & 6,61 & 0,73 & 9,70 & 0,09 & 2,21 & 5,06 \\
\hline & 150 & 7,76 & 0,64 & 8,85 & 0,14 & 2,00 & 4,32 \\
\hline \multirow{6}{*}{$\begin{array}{c}1 \\
5-10 \mathrm{~cm}\end{array}$} & 25 & 1,70 & 0,61 & 21,38 & 0,12 & 2,00 & 19,87 \\
\hline & 50 & 1,82 & 0,65 & 19,14 & 0,09 & 2,09 & 17,22 \\
\hline & 75 & 2,24 & 0,77 & 18,54 & 0,15 & 2,18 & 14,92 \\
\hline & 100 & 3,55 & 0,63 & 13,04 & 0,13 & 1,93 & 9,01 \\
\hline & 125 & 6,06 & 0,62 & 10,84 & 0,13 & 2,06 & 5,93 \\
\hline & 150 & 7,19 & 0,69 & 9,67 & 0,16 & 2,03 & 4,87 \\
\hline \multirow{6}{*}{$\begin{array}{c}1 \\
10-15 \\
\mathrm{~cm}\end{array}$} & 25 & 1,44 & 0,61 & 22,88 & 0,14 & 2,06 & 22,01 \\
\hline & 50 & 1,57 & 0,74 & 22,06 & 0,16 & 2,03 & 19,62 \\
\hline & 75 & 2,03 & 0,73 & 20,28 & 0,11 & 2,15 & 17,27 \\
\hline & 100 & 3,08 & 0,74 & 14,37 & 0,11 & 1,91 & 10,40 \\
\hline & 125 & 5,25 & 0,77 & 13,32 & 0,10 & 1,92 & 7,68 \\
\hline & 150 & 6,74 & 0,66 & 11,70 & 0,12 & 1,90 & 6,09 \\
\hline \multirow{6}{*}{$\stackrel{2}{2} 0-5 \mathrm{~cm}$} & 25 & 2,72 & 0,14 & 28,52 & 0,07 & 2,58 & 23,50 \\
\hline & 50 & 3,08 & 0,13 & 27,67 & 0,09 & 2,73 & 21,82 \\
\hline & 75 & 3,65 & 0,17 & 21,49 & 0,08 & 2,78 & 15,58 \\
\hline & 100 & 5,89 & 0,15 & 16,87 & 0,08 & 2,59 & 9,35 \\
\hline & 125 & 6,92 & 0,15 & 15,62 & 0,09 & 2,68 & 8,13 \\
\hline & 150 & 8,15 & 0,17 & 12,77 & 0,07 & 2,78 & 6,25 \\
\hline \multirow{6}{*}{$\stackrel{2}{2} \mathrm{~cm}$} & 25 & 2,22 & 0,16 & 33,47 & 0,06 & 2,40 & 30,70 \\
\hline & 50 & 2,37 & 0,12 & 32,28 & 0,05 & 2,47 & 28,72 \\
\hline & 75 & 3,32 & 0,15 & 26,89 & 0,05 & 2,69 & 20,42 \\
\hline & 100 & 5,22 & 0,16 & 17,04 & 0,05 & 2,51 & 10,82 \\
\hline & 125 & 6,18 & 0,16 & 16,67 & 0,07 & 2,93 & 9,37 \\
\hline & 150 & 6,71 & 0,15 & 15,88 & 0,06 & 3,16 & 8,57 \\
\hline \multirow{6}{*}{$\begin{array}{c}2 \\
10-15 \\
\mathrm{~cm}\end{array}$} & 25 & 2,03 & 0,14 & 36,14 & 0,06 & 2,38 & 34,66 \\
\hline & 50 & 2,11 & 0,14 & 34,06 & 0,05 & 2,45 & 32,15 \\
\hline & 75 & 3,10 & 0,16 & 31,44 & 0,07 & 2,66 & 24,64 \\
\hline & 100 & 4,31 & 0,16 & 19,27 & 0,07 & 2,61 & 12,87 \\
\hline & 125 & 5,49 & 0,15 & 18,05 & 0,07 & 2,67 & 10,73 \\
\hline & 25 & 2,03 & 0,14 & 36,14 & 0,06 & 2,38 & 34,66 \\
\hline
\end{tabular}

(1)G Niveis da necessidade de gesso; (2)CE condutividade elétrica e ${ }^{(3)}$ RAS relação de adsorção de sódio 
A.

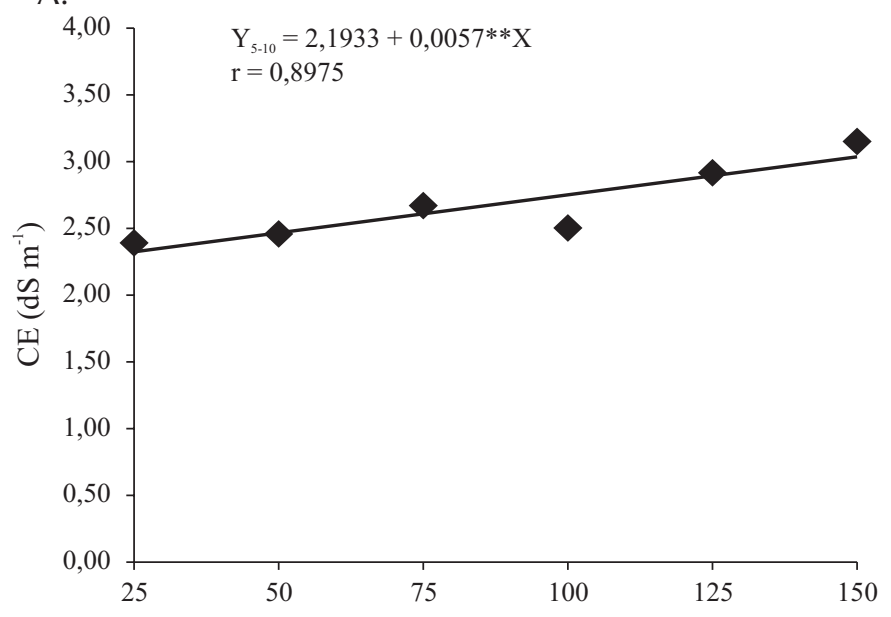

B.

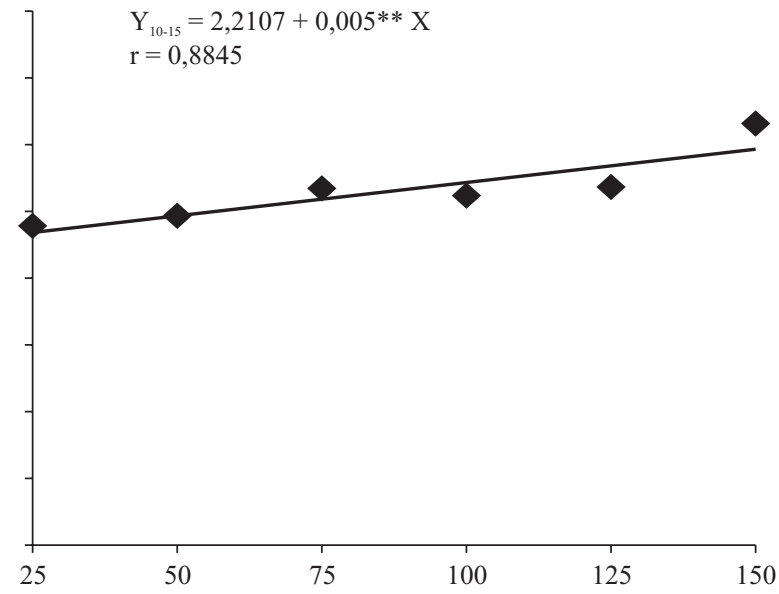

Níveis da necessidade de gesso (\%)

Figura 1. Condutividade elétrica do solo 2, em função dos níveis da necessidade de gesso, para as profundidades de 5 a $10 \mathrm{~cm}(\mathrm{~A})$ e de 10 a $15 \mathrm{~cm}(\mathrm{~B})$

A.
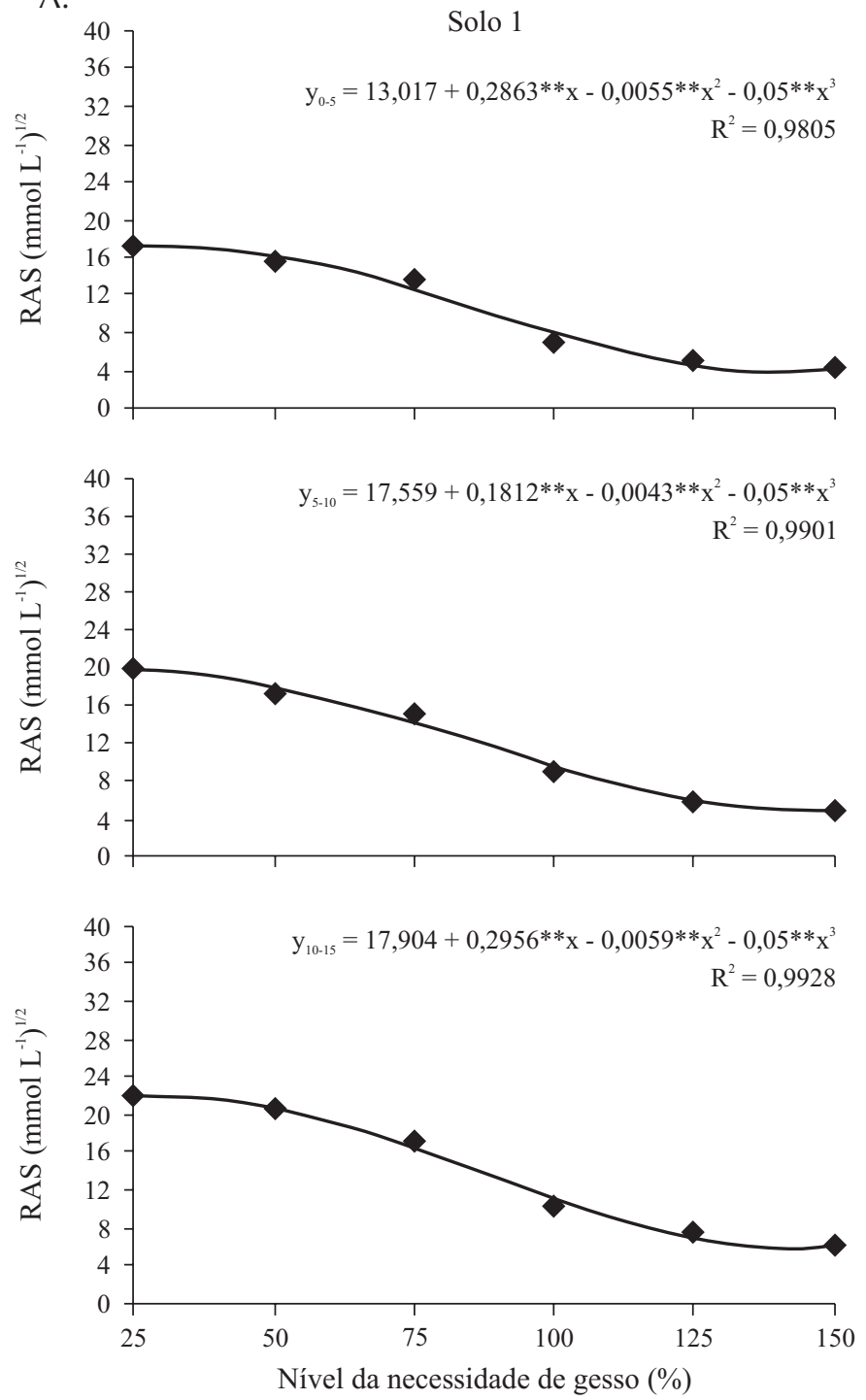

B.
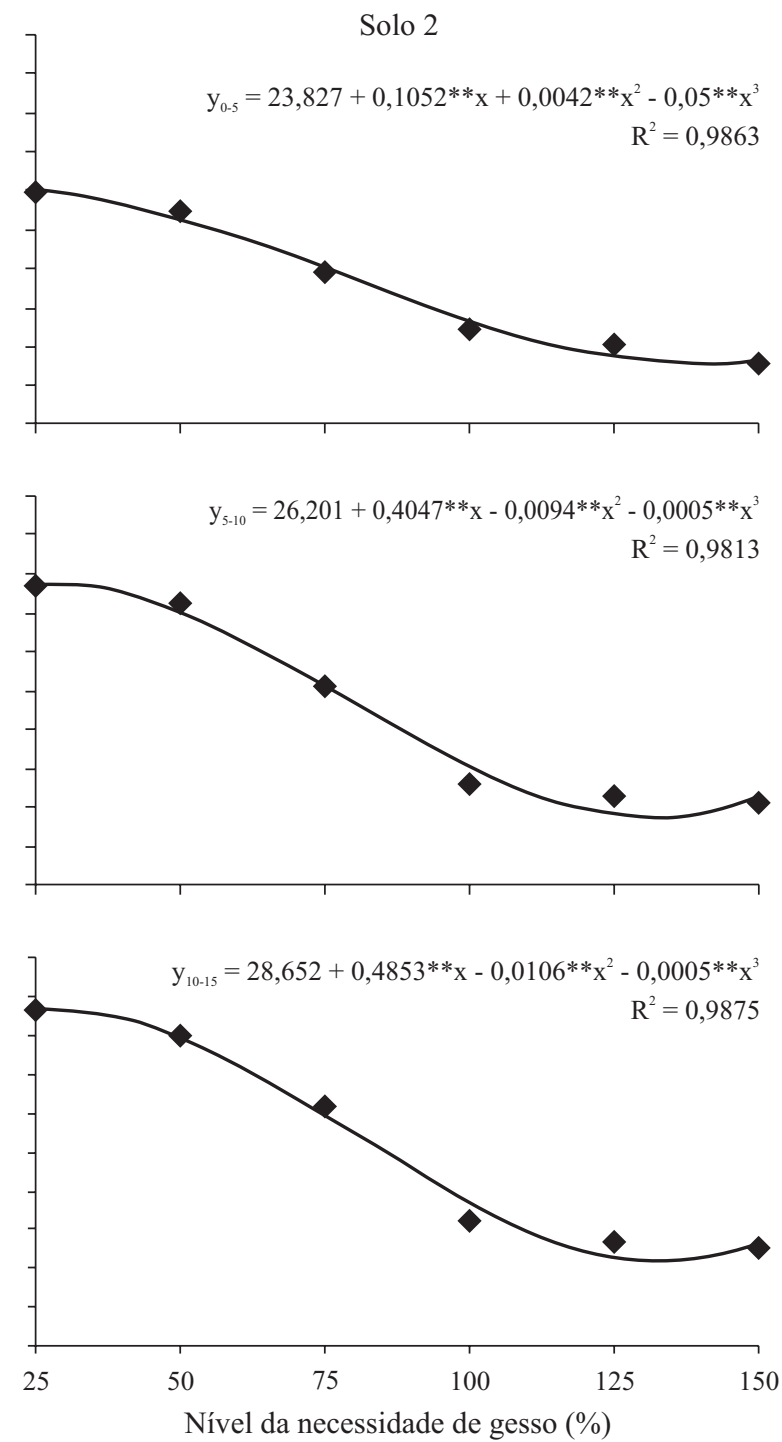

**Significativo a nível de $1 \%$

Figura 2. Relação entre a RAS e os níveis de necessidade de gesso para o solo 1 (A) e 2 (B) 
estão de acordo com Silveira (2000) que, aplicando gesso via água de irrigação em solos salino-sódicos de Custódia, nas profundidades de 0-20 e 20-50 cm, observou menor redução do sódio trocável para as maiores profundidades.

A relação entre os níveis da necessidade de gesso e a CE foi testada. Para o solo 01 não se verificou correlação entre as variáveis, mas para o solo 02 ocorreu diferença significativa para as profundidades de $5-10 \mathrm{~cm}$ e $10-15 \mathrm{~cm}$ (Figura 1) fato explicado porque os sais, que estavam na primeira camada foram, provavelmente, carreados para as camadas subseqüentes, favorecendo uma concentração maior de sais nessas profundidades. Rahman et al. (1996) constataram redução da condutividade elétrica de um solo salino-sódico para valores menores que $4 \mathrm{dS} \mathrm{m}^{-1}$ nas profundidades de $0-30 \mathrm{~cm}$ e de 30-60 cm, com a aplicação de gesso e posterior aplicação de uma lâmina de lixiviação.

Ocorreu diminuição da RAS dos solos estudados em todos os tratamentos, resultante das trocas iônicas entre o sódio e o cálcio, em todas as profundidades (Figura 2). Os tratamentos de 25,50 e $75 \%$ da necessidade de gesso não reduziram a RAS ao valor limite indicado por Barros et al. (2005) para correção da sodicidade dos solos (RAS > 13); entretanto, os tratamentos de 100, 125 e $150 \%$ da necessidade de gesso reduziram a RAS a níveis inferiores a este limite, em todas as profundidades, indicando a correção desses solos à sodicidade.

Em relação às camadas, a medida em que a profundidade aumentava a RAS também apresentava valores mais altos. A quantidade de corretivo que atingia as últimas camadas era menor que a da camada superior devido as substituições iônicas ocorridas na primeira camada ocasionando maior presença de sódio nas camadas inferiores. $\mathrm{O}$ efeito do gesso na redução da RAS também foi constatado por Barros et al. (2004), aplicando $100 \%$ da NG em quatro solos salino-sódicos de Pernambuco. Os autores observaram que o gesso diminuiu a RAS porém esta aumentava com a profundidade do solo.

\section{CONCLUSÕES}

1. A eficiência do gesso na correção da sodicidade do solo mostrou-se eficaz quando utilizado o nível de $100 \%$ da necessidade de gesso dos solos estudados.

2. A aplicação dos níveis de 100, 125 e $150 \%$ da necessidade de gesso mostrou-se eficiente na diminuição da RAS do extrato de saturação dos solos, reduzindo os teores de sódio dos solos.

\section{LITERATURA CITADA}

Barros, M. de F. C.; Fontes, M. P. F.; Alvarez, V. H.; Ruiz, H. A. Recuperação de solos afetados por sais pela aplicação de gesso de jazida e calcário no Nordeste do Brasil. Revista Brasileira de Engenharia Agrícola e Ambiental, v.8, n.1, p.59-64, 2004.

Barros, M. de F. C.; Fontes, M. P. F.; Alvarez, V. H.; Ruiz, H. A. Aplicação de gesso e calcário na recuperação de solos salinosódicos do estado de Pernambuco. Revista Brasileira de Engenharia Agrícola e Ambiental, v.9, n.3, p.320-326, 2005.

Barros, M. de F. C.; Magalhães, A. F. Avaliações de métodos de determinação da necessidade de gesso em solos salinosódicos. Revista Brasileira de Ciência do Solo, v.13, p.119123, 1989.

EMBRAPA - Empresa Brasileira de Pesquisa Agropecuária. Manual de métodos de análises de solo. 2.ed. Rio de Janeiro: EMBRAPA, 1997. 212p.

Gheyi, H. R.; Azevedo, N. C.; Batista, M. A. F.; Santos, J. G. R. Comparação de métodos na recuperação de solo salinosódicos. Revista Brasileira de Ciência do Solo, v.19, p.173178, 1995.

Magalhães, A. F. Métodos de análises químicas para solos salinos e sódicos. Recife: Departamento de Agronomia/UFRPE, 1987. 30p.

Nascimento, C. W. A.; Barros, M. de F. C. Utilização do gesso na agricultura. Curso de gestão ambiental e otimização da exploração e utilização do gesso na região do Araripe - PE. Recife: UFRPE, 2003, 22p.

Oliveira, L. B. de; Rosas, R. M.; Ferreira, M.da G. de V. X.; Lima, J. F. W. F. de; Marques, F. A. Interferências pedológicas aplicadas ao perímetro irrigado de Custódia, PE. Pesquisa Agropecuária Brasileira, v.37, n.10, p.1477-1486, 2002.

Rahman, A. H. A.; Dahab, M. H.; Mustafa, M. A. Impact of soil amendments on intermittent evaporation, moisture distribuition and salt redistribuition in saline-sodic clay soil columns. Soil Science, v.161, p.793-802, 1996.

Richards, L. A. Diagnosis and improvement of saline and alkali soils. Washington: U.S. Department of Agriculture, 1954, 160p. Handbook 60

Schoonover, W. R. Examination of soils for alkali. Berkeley: University of California. Extension Service, 1952. Mimeographed

Silveira, K. R. Influência da aplicação de gesso na água de irrigação sobre a salinidade e condutividade hidráulica de solos aluviais. Recife: UFRPE, 2000. 73p. Dissertação Mestrado 